\title{
Qualitative Research: A way of Listening to Patients
}

\author{
Sailaj Ranjitkar \\ Msc (Health and Science); University of Central \\ Lanchanshire, UK
}

Research can be quantitative or qualitative. Quantitative research helps to prove or disprove a hypothesis by using numerical data and quantifying the problem. Qualitative research however helps to understand patient problems better, whether it is physical, emotional or psychological. An example of the importance of qualitative research is to investigate the reason for non-adherence to treatment like tuberculosis (Munro et al., 2007). It has been seen that nearly $50 \%$ of patients are not adherent to tuberculosis treatment (Cuneo \& Snider, 1989). Therefore, it is important to understand if the patients are aware of it being a life threatening condition but curable and to understand the reason of non-adherence even if they knew the consequences. The time has come to involve patients and their caregivers in health care research than just having passive participation as done traditionally (Phoenix et al., 2018). Patient engagement has been seen to have a definite advantage especially for retaining them in the research (Domecq et al., 2014; Edwards et al., 2011) but there is no definite evidence for any particular strategy of partnership (Domecq et al., 2014).

Qualitative research has been in practice to explore the opinions and thoughts of people with underlying problems. This can help to develop a hypothesis for quantitative research, which in return helps to quantify the problem numerically. The five methods of qualitative research are ethnography, narrative, case study, phenomenological and grounded theory (Creswell, 2013), all of them collecting data via interviews, observations and text review except Ethnography where the researcher is part of the study and experiences the environment him/herself. The researcher may even live in the environment and follow the participants for years. This method is appropriate to study culture of a society and assess if culture, rituals or social practices are responsible for making certain decisions like any one sex of the society receiving treatment earlier than the other.

Narrative method consists of interviewing one or two people and hearing their views in detail over a long period of time, even years. This is useful to understand a persons' daily activity. An example is to understand how a patient with glaucoma, retinopathy or any chronic condition is coping with life on a daily basis.

Case study methodology was brought into limelight by Harvard Business School. It is a way to study an organisation, event or a company using various sources of data to explain or describe an event.

Phenomenological method is a way to describe a phenomenon, for example there may be a sudden increase in Trachoma patients in certain sections of society over few years 
despite the governments' effort to control it. It may be necessary to understand this phenomenon by interviewing patients to describe this change. Grounded theory on the other hand tries to give a reason for the event. This is usually done by first reading all the interviews and trying to understand their meaning. They are then reflected upon and coded into groups or categories. These categories are then assessed for common factors whether there are agreements or disagreements. Finally their inter-rater or intra-rater reliability can be checked using Kappa coefficient.

Each of these methods has their own pros and cons but grounded theory is a method well accepted in social science and academic research. The difficulty however is the amount of time it consumes to collect the data, also considering the larger sample size necessary compared to the other four methods of qualitative research.

This method is in vogue especially in 'Psychology' and 'Nursing and Healthcare' and is gradually being utilised in other fields of medicine too. We as doctors discuss the success of a treatment or surgery, which is usually considered from the perspective of the treatment giver and not from the perspective of the receiver. That is probably the reason 'Patient reported outcome measures' (PROM) are being utilized more often. This method is important in every field of medicine and Ophthalmology in Nepal is no exception not to venture into patient based research, especially considering the variation in patient satisfaction. It is therefore time to listen to our patients and organise a patient-centred approach whether it be research or management of diseases.

\section{References}

Creswell, J.W. (2013). Qualitative enquiry and research design: Choosing among five approaches (Third edition), 1-472.

Cuneo, W.D., Snider, D.E. (1989). Enhancing patient compliance with tuberculosis therapy. Clin Chest Med, 10, 375-380.

Domecq, P.J., Prutsky, G., Elraiyah, T., Wang, Z., Nabhan, M., Shippee, N. (2014). Patient engagement in research: a systematic review. BMC Health Serv Res, 14, 89.

Edwards, V., Wyatt, K., Logan, S., Britten, N. (2011). Consulting parents about the design of a randomized controlled trial of osteopathy for children with cerebral palsy. Health Expect,14(4),429-38.

Munro, S.A., Lewin, S.A., Smith, H.J., Engel, M.E., Fretheim, A. (2007). Patient adherence to tuberculosis treatment: A systematic review of qualitative research. PLoS Med, 4, e238.

Phoenix, M., Nguyen, T., Gentles, S.J., VanderKaay, S., Cross, A., Nguyen, L. (2018). Using qualitative research perspectives to inform patient engagement in research. Research Involvement and Engagement, 4(20), 1-5. https://doi.org/10.1186/ s40900-018-0107-1 